since $(m-1)^{2}$ is the number of apparent intersections of $C^{m}$ and $C^{m-1}$. If we replace $m$ by $(n+1) / 2$, the result is

$$
h=(n-1)^{2} / 4+h^{\prime \prime}+h^{\prime \prime \prime}
$$

and hence

$$
P=(n-1)(n-3) / 4-h^{\prime \prime}-h^{\prime \prime \prime} .
$$

To find $h^{\prime}$ or $h^{\prime \prime}$ and $h^{\prime \prime \prime}$ we repeat the process. We soon arrive at the component curves whose orders are equal to or less than twice the dimensions of the several sub-spaces in which the component curves lie, and then apply Veronese's rule. Or, in case $n$ is very large, we arrive at the component curves which are all plane and have no double points.

It is to be noticed that $h^{\prime}=h^{\prime \prime}=h^{\prime \prime \prime}=0$ if $r=3$. Hence the greatest deficiency of any 3 -space curve $C^{n}$ is

and

$$
p=(n-2)^{2} / 4, \quad \text { for } n \text { even; }
$$

$$
p=(n-1)(n-3) / 4, \quad \text { for } n \text { odd. }
$$

The University of California

\title{
A NOTE ON CERTAIN CONTINUOUS NON-DIFFERENTIABLE FUNCTIONS*
}

BY F. W. PERKINS

This note gives a treatment of some phases of the theory of a class of functions of which a particular example has already been studied by the author in a note entitled $A n$ elementary example of a continuous non-differentiable function, in the American Mathematical Monthly (vol. 34 (1927), pp. 476-478). The method there used for the construction of a function with the desired properties bears some resemblance to that used by Brodén, Köpke, and Steinitz for the

* Presented to the Society, September 6, 1928. 
construction and study of functions with notable properties with respect to differentiability.* The example consists of a function belonging to a class investigated by Steinitz, but the discussion is somewhat more elementary in form than that given previously in the more general treatment. The method consists, briefly, in assigning functional values at an everywhere dense set of points in such a way that the function can be extended so as to be continuous on the whole interval and to have no derivative. The principle involved enables us not only to construct non-differentiable functions but also to give in simple form a sufficient condition that a continuous function be convertible into a non-differentiable continuous function by a certain change of variable.

First let us examine a few of the more immediate consequences of non-differentiability. We note that such a function cannot be of bounded variation on any subinterval, for by a well known theorem, a function of bounded variation has a derivative almost everywhere, that is, except possibly at the points of a set of measure zero in the sense of Lebesgue. $\dagger$ It follows that a function which has no derivative must have maxima and minima $\ddagger$ on every subinterval.

* E. Steinitz, Stetigkeit und Differentialquotient, Mathematische Annalen, vol. 52 (1899), pp. 58-69. For a description of the methods of Brodén and Köpke, see Hobson, The Theory of Functions of a Real Variable, 3d ed., 1927, vol. 1, pp. 387 ff. See also a paper by E. H. Moore, Transactions of this Society, vol. 1 (1900), pp. $81 \mathrm{ff}$; and one by Hahn, Jahresbericht der Vereinigung, vol. 26 (1918), pp. $281 \mathrm{ff}$.

$\dagger$ See, for example, Carathéodory, Vorlesungen über Reelle Funkiionen, 2d ed., 1927, p. 188, Satz 9, and p. 563 ff.

$\ddagger$ By a maximum value of a function we shall mean a value attained at a point $x^{\prime}$, not exceeded at any point in the neighborhood of $x^{\prime}$, and not maintained constantly in any interval having $x^{\prime}$ as an interior or end point. The corresponding definition for a minimum is of course also used. It may be noted that a function may have an extremum in this sense which is not an extremum in the "strict" sense in which it is required that a maximum value, for example, be actually greater than all other functional values in the neighborhood of the point at which it is attained. Consider, for instance, the behavior at $x=0$ of the function defined below:

$$
\left\{\begin{array}{l}
f(x)=0 \text { when } x=0 \\
f(x)=-x^{2} \sin ^{2}(1 / x) \text { when } 0<|x|<1 .
\end{array}\right.
$$


This condition may be expressed analytically in the following manner: Let $f(x)$ be any function continuous on the interval $I: a \leqq x \leqq b$, but not constant on any subinterval of $I$. A necessary and sufficient condition that $f(x)$ have maxima and minima which are everywhere dense on $I$ is that every subinterval $x_{0} \leqq x \leqq x_{3}$ at the ends of which $f(x)$ assumes distinct values contains two interior points $x_{1}$ and $x_{2}$ such that $x_{1}<x_{2}$ and

$$
\frac{f\left(x_{1}\right)-f\left(x_{2}\right)}{f\left(x_{3}\right)-f\left(x_{0}\right)}>0 .
$$

For if $f(x)$ has extrema which are everywhere dense we may obviously choose $x_{1}$ and $x_{2}$ so that the numerator has the same sign as the denominator. On the other hand, if the fraction is positive, the function $f(x)$ has an extremum between $x_{0}$ and $x_{3}$. In establishing this fact, we may assume, without loss of generality, that the denominator is positive. In the case in which $f\left(x_{0}\right) \leqq f\left(x_{2}\right)$, we see that $f(x)$ has a maximum between $x_{0}$ and $x_{2}$; if $f\left(x_{0}\right)>f\left(x_{2}\right)$, we infer that $f(x)$ has a minimum between $x_{1}$ and $x_{3}$. Moreover, on any subinterval at the ends of which $f(x)$ assumes equal values, $f(x)$ must have an extremum at an interior point. It follows, then, that $f(x)$ has maxima and minima which are everywhere dense on $I$.

We note that this property in regard to extrema is one which is preserved if we replace the variable $x$ by a variable $\xi$ connected with it by the relation $x=\phi(\xi)$, where the function $\phi(\xi)$ is continuous on the interval $a \leqq \xi \leqq b$ and satisfies the conditions $\phi\left(\xi^{\prime}\right)<\phi\left(\xi^{\prime \prime}\right)$ if $\xi^{\prime}<\xi^{\prime \prime}, \phi(a)=a, \phi(b)=b$. The property of non-differentiability is obviously not always preserved by such a change of variable. Hence we see that although the condition that the extrema of $f(x)$ be everywhere dense on $I$ is necessary for non-differentiability, it is not sufficient. Moreover there exist functions with extrema which are everywhere dense and which cannot be rendered non-differentiable by such a change of variable. 
We propose to show now that a slight modification of the condition given above for everywhere dense extrema leads to a condition which is sufficient to insure non-differentiability after a suitable change of variable.

TheOREM. If $f(x)$ is continuous on the interval $I: a \leqq x \leqq b$, but is not constant on any subinterval, and if there exists a positive quantity $\delta$ such that every subinterval $x_{0} \leqq x \leqq x_{3}$ at the ends of which $f(x)$ assumes distinct values contains two points $x_{1}$ and $x_{2}$ such that $x_{1}<x_{2}$ and

$$
\frac{f\left(x_{1}\right)-f\left(x_{2}\right)}{f\left(x_{3}\right)-f\left(x_{0}\right)}>\delta,
$$

then there exists a function $\phi(\xi)$, continuous and monotonically increasing on the interval $a \leqq \xi \leqq b$, such that $F(\xi)=f[\phi(\xi)]$ fails to have a derivative with respect to $\xi$ at every point of the interval $a \leqq \xi \leqq b$.

We shall first treat the case in which $f(x)$ attains its least and greatest values on $I$ at the points $x=a$ and $x=b$ respectively: $f(a) \leqq f(x) \leqq f(b)$ or $f(b) \leqq f(x) \leqq f(a)$ when $a \leqq x \leqq b$.

We consider first the value of the fraction

$$
\frac{f\left(x_{1}\right)-f\left(x_{2}\right)}{f(b)-f(a)}
$$

for values of $x_{1}$ and $x_{2}$ such that $a \leqq x_{1} \leqq x_{2} \leqq b$. Since this is a particular form of the fraction given in the hypothesis of the theorem, we know that its greatest value must be greater than $\delta$. We note that to attain this maximum value we may have neither $x_{1}=a$ nor $x_{2}=b$. The fraction may conceivably attain its maximum value at more than one pair of points $x_{1}$ and $x_{2}$. However, the set of all points which may be used as $x_{1}$ is closed, and therefore contains a point farthest to the left. We shall call this point $X_{1}$. All the points which are possible choices for an $x_{2}$ corresponding to $x_{1}=X_{1}$ form a closed set, and therefore contain a point farthest to the right, which we shall call $X_{2}$. We now repeat the same process for each of the intervals $a \leqq x \leqq X_{1}$, 
$X_{1} \leqq x \leqq X_{2}$ and $X_{2} \leqq x \leqq b$. It will be noted that the function $f(x)$ attains its extreme values on any one of these intervals at the ends of that interval. The three new pairs of points thus obtained, taken together with the points $x=X_{1}$ and $x=X_{2}$, divide the interval $I$ into nine parts, to each of which we apply the process again, and so on indefinitely.

The set $\{X\}$ of all points thus obtained is everywhere dense on $I$. For if this is not true there exists a subinterval $\gamma$ containing none of these points. Let $\mu$ be the difference between the greatest value and the least value of $f(x)$ on $\gamma$. Since $f(x)$ is not constant on any interval we know that $\mu>0$. Let $I_{1}$ be that subinterval obtained by the first subdivision (by the points $X_{1}$ and $X_{2}$ ) which contains $\gamma$; let $I_{2}$ be that subinterval obtained by the second subdivision which contains $\gamma$, and so on. In this way we get an infinite sequence of subintervals $I_{1}, I_{2}, I_{3}, \cdots$, each of which contains all the following, and also contains $\gamma$. Let $X_{1}, X_{1}^{\prime}$, $X_{1}^{\prime \prime}, \ldots$, be the left hand end points of these intervals. Each of these points lies to the left of the next following point of the sequence, and also to the left of $\gamma$. Consequently they have a unique limit point, $X$, on $I$. Moreover, at any two successive points of the sequence $X_{1}, X_{1}^{\prime}, X_{1}^{\prime \prime}, \cdots$, the function $f(x)$ has values which, it is readily seen, differ by at least $\mu$. This implies that $f(x)$ is discontinuous at $x=X$, contrary to our hypothesis. This contradiction establishes the desired result. It may be noted, incidentally, that the points of the everywhere dense set which we have just described yield extrema of the function $f(x)$.

We now proceed to the definition of the function $\phi(\xi)$. Since this function is to be continuous it is possible to define it completely by assigning suitable functional values at the points of an everywhere dense set. First we set $\phi(a)=a$, $\phi(b)=b$, and $\phi\left(\xi_{1}\right)=X_{1}$ and $\phi\left(\xi_{2}\right)=X_{2}$, where*

* Since $F(\xi)=f[\phi(\xi)]$, while $\phi(a)=a$ and $\phi(b)=b$, it follows that $F(a)=f(a)$, and $F(b)=f(b)$. However in analogous work a little later it will prove necessary to distinguish carefully between the functions $F$ and $f$. 


$$
\left\{\begin{array}{l}
\xi_{1}=\frac{a F(b)-b F(a)+f\left(X_{2}\right)[b-a]}{F(b)-F(a)} \\
\xi_{2}=\frac{a F(b)-b F(a)+f\left(X_{1}\right)[b-a]}{F(b)-F(a)}
\end{array}\right.
$$

These formulas may be given a simple geometric interpretation: $\xi_{1}$ and $\xi_{2}$ are the abscissae of the points of intersection of the lines joining $[a, F(a)]$ and $[b, F(b)]$ and the lines $\eta=f\left(X_{2}\right)$ and $\eta=f\left(X_{1}\right)$, respectively, in the $\xi, \eta$ plane.

Each of the subintervals into which $X_{1}$ and $X_{2}$ divide $I$ contains two points standing in the same relation to that subinterval as $X_{1}$ and $X_{2}$ do to $I$. By the method given above we determine the corresponding values of $\xi$. We repeat this process indefinitely, obtaining an infinite set of points at each of which the function $\phi(\xi)$ is defined. The order relations between these points is the same as the order relations between the corresponding points $X$, and so $\phi(\xi)$, in so far as it is now defined, is a monotonically increasing function.

The straight line joining $[a, F(a)]$ and $[b, F(b)]$ is the graph of a function $F_{0}(\xi)$ which is a first approximation to the function $F(\xi)$. The broken line joining the points $[a, F(a)]$, $\left[\xi_{1}, F\left(\xi_{1}\right)\right],\left[\xi_{2}, F\left(\xi_{2}\right)\right]$ and $[b, F(b)]$ yields a second approximation, $F_{1}(\xi)$; a third approximation may be obtained by replacing each segment of this line by a broken line of three pieces related to the segment in the same way as the graph of the second approximation is to that of the first, and so on.*

Denoting by $\lambda_{i}$ the slope of a line segment of the graph of an approximating function $F_{i}(\xi)$, we see, by a simple computation, that the slope of the second of the line segments by which it is replaced in the graph of $F_{i+1}(\xi)$ is $-\lambda_{i}$, while the slope of each of the other two segments is at least as great numerically as $\left|\lambda_{i}\right|(1+\delta)$. This enables us to prove that the

* The sequence $F_{0}(\xi), F_{1}(\xi), F_{2}(\xi), \cdots$ converges uniformly, but this is of no importance for our proof, as we shall obtain $F(\xi)$ by another method. We shall have occasion, however, to use these functions in another connection. 
point set on which we have already defined the function $\phi(\xi)$ is everywhere dense. For if such is not the case there exists an interval throughout which each approximating function is linear with a slope at least as great numerically as the positive quantity $\left|\lambda_{0}\right|$. Consequently there exists a number $c>0$ and pairs of points of the set $\{X\}$, as near together as we choose to stipulate in advance, at which the function $f(x)$ assumes values differing by at least $c$. But this contradicts the hypothesis of continuity, and so shows that $\phi(\xi)$ is defined on an everywhere dense set of points.

At any point $\xi=\xi^{\prime}$ at which $\phi(\xi)$ is not already defined we set $\phi(\xi)$ equal to the least upper bound of all its known values on the interval $a \leqq \xi<\xi^{\prime}$. The function $\phi(\xi)$ is now defined and monotonically increasing on the interval $a \leqq \xi \leqq b$. It is continuous there; for a discontinuity could only be a finite jump, which would imply the existence of a subinterval of $I$ free from points of the set $\{X\}$, contrary to the known fact that this set is everywhere dense.

We shall now show that the continuous function $F(\xi)=f[\phi(\xi)]$ has no derivative at any point of the interval $a \leqq \xi \leqq b$. We understand this statement to mean that at no point $\xi=\xi^{\prime}$ of this interval does the difference quotient $\left[F\left(\xi^{\prime}+h\right)-F\left(\xi^{\prime}\right)\right] / h$ approach a limit as $h$ approaches zero.*

If $\xi^{\prime}$ corresponds to one of the points of the set $\{X\}$, then it is clear that $F(\xi)$ has no derivative at $\xi=\xi^{\prime}$, because, for sufficiently large values of $n$, the forward derivative of $F_{n+1}(\xi)$ at that point is at least $1+\delta$ times as great numerically as the forward derivative of $F_{n}(\xi)$ at $\xi=\xi^{\prime}$, and so the forward derivative of $F_{n+k}(\xi)$ at $\xi=\xi^{\prime}$ can be made as large numerically as we like by choosing $k$ sufficiently large. Since the forward derivatives of $F_{n}(\xi), F_{n+1}(\xi)$, $F_{n+2}(\xi), \cdots$ at $\xi=\xi^{\prime}$ give us the values of the difference quotient $\left[F\left(\xi^{\prime}+h\right)-F\left(\xi^{\prime}\right)\right] / h$ for a sequence of values of $h$ approaching zero as a limit, we see that the difference

* Some writers use a definition of differentiability which allows the difference quotient either to approach a limit or to become positively or negatively infinite. 
quotient cannot approach a limit in this case. Backward derivatives of the approximating functions may be used equally well. The forward and backward derivatives have opposite signs, and so we see that for a certain sequence of positive values of $h$ tending toward zero the difference quotient becomes infinite with one sign, while for a certain sequence of negative values of $h$ tending toward zero the difference quotient becomes infinite with the other sign. It should be noted, however, that this does not necessarily mean in either case that the difference quotient becomes infinite independently of the manner in which $h$ approaches zero from one side.*

It may also be noted that $F(\xi)$ has no forward derivative at the point $x=a$ and no backward derivative at $x=b$, though the difference quotient may conceivably become positively or negatively infinite at these points.

It remains to show that $F(\xi)$ has no derivative at any point $\xi^{\prime}$ of the open interval $a<\xi<b$ which does not correspond to a point of the set $\{X\}$. At such a point each of the functions $F_{n}(\xi)$ has a derivative. Let $\xi=\xi_{n}^{\prime \prime}<\xi^{\prime}$ and $\xi=\xi_{n}^{\prime \prime \prime}>\xi^{\prime}$ be points corresponding to points of the set $\{X\}$ between which $F_{n}(\xi)$ has a constant derivative. If we set $h=h_{n}^{\prime \prime}=\xi_{n}^{\prime \prime}-\xi^{\prime}$ or $h=h_{n}^{\prime \prime \prime}=\xi_{n}^{\prime \prime \prime}-\xi^{\prime}$, the value obtained for the difference quotient $\left[F\left(\xi^{\prime}+h\right)-F\left(\xi^{\prime}\right)\right] / h$ is in one case not greater algebraically than $D_{\xi} F_{n}\left(\xi^{\prime}\right)$ and in the other case not less algebraically than $D_{\xi} F_{n}\left(\xi^{\prime}\right)$. Since $\lim h_{n}^{\prime \prime}=\lim {h_{n}}^{\prime \prime \prime}=0$, it

* In special cases, however, this may happen. In the case of the function previously studied by the author, cited above, it can readily be shown that at each of the everywhere dense set of maxima (given by $x=p / 3^{n}$, where $n$ is any positive integer and $p$ any odd positive integer less than $3^{n}$ ) the difference quotient becomes positively infinite on the left and negatively infinite on the right, while at the minima (given by the remaining values of $x$ of the form $p / 3^{n}$ in the open interval $0<x<1$ ) the difference quotient becomes negatively infinite on the left and positively infinite on the right. For $x=0$ and $x=1$ the difference quotient becomes positively infinite. The coordinates of the extrema are here given for the function as defined in the earlier paper. It is readily verified that the properties stated regarding the behavior of the difference quotient are preserved (in this case) by the transformation described in the present paper. 
follows that this difference quotient can approach a limit only if $D_{\xi} F_{n}\left(\xi^{\prime}\right)$ approaches a limit as $n$ becomes infinite. This, however, is never the case. In the ratio $D_{\xi} F_{n+1}\left(\xi^{\prime}\right) / D_{\xi} F_{n}\left(\xi^{\prime}\right)$ neither the numerator nor the denominator is zero and neither approaches zero as a limit. Moreover for any $n$ this ratio has either the value -1 or else is greater than $1+\delta$, and so cannot approach the limit unity, as it would if $D_{\xi} F_{n}\left(\xi^{\prime}\right)$ approached a limit. Hence the difference quotient $\left[F\left(\xi^{\prime}+h\right)-F\left(\xi^{\prime}\right)\right] / h$ cannot approach a limit.

This completes the proof of the theorem for the case in which either $f(a) \leqq f(x) \leqq f(b)$ or $f(b) \leqq f(x) \leqq f(a)$. The proof can be extended to the general case immediately by dividing the interval $I$ into a finite or denumerably infinite set of subintervals, each of which satisfies the above condition, and then applying the above reasoning to each such subinterval. This subdivision of $I$ can be accomplished by choosing as the first subinterval the interval between points at which $f(x)$ attains its extreme values. In case these values are attained at more than one pair of points we may choose one particular pair by means of the device used in the similar situation for the choice of $X_{1}$ and $X_{2}$. We then apply this method to the remaining interval or intervals and continue the process indefinitely, or until we have exhausted $I$.

Dartmouth College 